\title{
Contribuições da metodologia construtivo- interpretativa na pesquisa sobre o desenvolvimento da subjetividade
}

\author{
Maristela Rossato \& Albertina Mitjáns Martínez
}

\begin{abstract}
Resumo
O objetivo do artigo é apresentar e analisar a metodologia construtivointerpretativa utilizada em uma pesquisa sobre o desenvolvimento da subjetividade. A Epistemologia Qualitativa desenvolvida por González Rey para o estudo da subjetividade, numa perspectiva cultural-histórica, é a base constitutiva dessa metodologia, pois concebe a produção do conhecimento como um processo de comunicação e diálogo, legitima a contribuição do caso singular na produção do conhecimento científico e concebe o conhecimento como um processo construtivointerpretativo. A metodologia construtivo-interpretativa constituiu-se num contínuo processo de interpretação e construção do conhecimento a partir das informações produzidas na realização dos instrumentos que tiveram a função de constituírem-se favorecedores da expressão dos participantes. A ação ativa, criativa e imaginativa do pesquisador possibilitou a mobilidade entre sua base teórica e as informações no processo da pesquisa. O processo de análise das informações produzidas foi realizado ao longo de toda a pesquisa, por meio da produção de indicadores que vieram a articular-se em hipóteses pela relevância que assumiram para o problema em discussão. Esse processo construtivo-interpretativa possibilitou gerar novos campos de inteligibilidade ao problema em estudo e novas zonas de sentido à base teórica do pesquisador. A pesquisa do desenvolvimento da subjetividade implicou em assumir e enfrentar o desafio de analisar fenômenos que estão em processos de mudança contínua.
\end{abstract}

Palavras-chave:

epistemologia qualitativa; metodologia construtivo-interpretativa; desenvolvimento da subjetividade. 


\title{
Contributions of constructive-interpretative methodology in research on the development of subjectivity
}

\begin{abstract}
The purpose of this article is to present and analyze the constructive-interpretative methodology used in a research on the development of subjectivity. The Qualitative Epistemology developed by González Rey for the study of subjectivity, in a cultural-historical perspective, is the constitutive basis of this methodology, since it conceives the production of knowledge as a process of communication and dialogue, legitimizes the contribution of the singular case in the production of knowledge and conceives knowledge as a constructive-interpretive process. The constructive-interpretative methodology was constituted in a continuous process of interpretation and construction of the knowledge based on the information produced through the application of instruments that had the function to facilitate the expression of the participants. The active, creative and imaginative action of the researcher allowed the mobility between its theoretical base and the information obtained in the research process. The process of analyzing the information produced throughout the research, results from the construction of indicators articulated in relevant hypotheses that are assumed for the problem under discussion. This constructive-interpretative process made it possible to generate new fields of intelligibility to the problem under study and new areas of meaning to the theoretical basis of the researcher. The research of the development of subjectivity implied in assuming and facing the challenge of analyzing phenomena that are in processes of continuous change.
\end{abstract}

Keywords: qualitative epistemology; constructive-interpretative methodology; development of subjectivity.

\section{Apports de méthodologie constructive-interprétative dans la recherche sur le développement de la subjectivité}

Résumé: Le but de cet article est de présenter et d'analyser la méthodologie constructive-interprétative utilisée dans une recherche sur le développement de la subjectivité. L'Épistémologie qualitative développée par González Rey pour l'étude de la subjectivité dans une perspective historique et culturelle est la base constitutive de cette méthodologie qui conçoit la production de connaissances en tant que processus de communication et de dialogue, légitimise la contribution du singulier dans la production du savoir et conçoit le savoir comme un processus d'interprétation constructive. La méthodologie constructive-interprétative a été constituée dans un processus continu d'interprétation et de construction de la connaissance basée sur l'information produite dans la réalisation des instruments qui avait pour fonction d'être favorable à l'expression des participants. L'action active, créative et imaginative du chercheur a permis la mobilité entre sa base théorique et l'information dans le processus de recherche. Le processus d'analyse de l'information produite a été réalisé tout au long de la recherche, à travers la production d'indicateurs qui ont été articulés dans des hypothèses en raison de la pertinence qu'ils ont prise pour le problème en discussion. Ce processus constructif d'interprétation permis de générer de nouveaux champs d'intelligibilité au problème étudié et de nouveaux domaines de sens à la base théorique du chercheur. La recherche du développement de la subjectivité implique assumer et relever le défi de l'analyse des phénomènes qui sont dans des processus de changement continu.

Mots-clés: épistémologie qualitative; méthodologie constructive-interprétative; développement de la subjectivité.

\section{Contribuciones de la metodología constructivo-interpretativa en la investigación sobre el desarrollo de la subjetividad}

Resumen: El objetivo del artículo es presentar y analizar la metodología constructivo-interpretativa utilizada en una investigación sobre el desarrollo de la subjetividad. La Epistemología Cualitativa desarrollada por González Rey para el estudio de la subjetividad, en una perspectiva cultural-histórica, es la base constitutiva de esa metodología, pues concibe la producción del conocimiento como un proceso de comunicación y diálogo, legitima la contribución del caso singular en la producción del conocimiento científico y concibe el conocimiento como un proceso constructivointerpretativo. La metodología constructivo-interpretativa se constituyó en un continuo proceso de interpretación y construcción del conocimiento a partir de las informaciones producidas en la realización de los instrumentos que tuvieron la función de constituirse favorecedores de la expresión de los participantes. La acción activa, creativa e imaginativa del investigador posibilitó la movilidad entre su base teórica y las informaciones en el proceso de la investigación. El proceso de análisis de las informaciones producidas fue realizado a lo largo de toda la investigación, a través de la producción de indicadores que vinieron a articularse en hipótesis por la relevancia que asumieron para el problema en discusión. Este proceso constructivo-interpretativo posibilitó generar nuevos campos de inteligibilidad al problema en estudio y nuevas zonas de sentido a la base teórica del investigador. La investigación del desarrollo de la subjetividad implicó en asumir y enfrentar el desafío de analizar fenómenos que están en procesos de cambio continuo.

Palabras Clave: epistemología cualitativa; metodología constructiva-interpretativa; desarrollo de la subjetividad. 


\section{Introdução}

Este artigo tem por objetivo apresentar e analisar a metodologia construtivo-interpretativa utilizada numa pesquisa sobre o desenvolvimento da subjetividade na trajetória de superação das dificuldades de aprendizagem escolar em estudantes dos anos iniciais do Ensino Fundamental (Rossato, 2009). Essa metodologia é fundamentada pelos pressupostos da Epistemologia Qualitativa, desenvolvida por González Rey (2002, 2003, 2005a, 2005b, 2011, 2015; González Rey \& Mitjáns Martínez, 2016, 2017) como recurso para a pesquisa da subjetividade, numa perspectiva cultural-histórica. Seu processo de construção iniciou diante do silêncio epistemológico identificado na história da Psicologia que esvaziou as possibilidades de uma unidade teoria-epistemologia-metodologia na produção do conhecimento (González Rey, 2013). Santos \& Menezes (2010), anteriormente, já chamavam a atenção para os problemas epistemológicos vividos pelas ciências, denunciando a dominação de modelos importados e distantes da realidade dos fenômenos estudados: "Por que razão, nos últimos dois séculos, dominou uma epistemologia que eliminou da reflexão epistemológica o contexto cultural e político da produção e reprodução do conhecimento?" (p. 11).

Para González Rey, desde o início de sua obra, fez-se necessário uma base epistemológico-metodológica que fosse coerente com a complexidade teórica desenvolvida em torno da subjetividade, a saber: (1) compreensão da subjetividade como sistema complexo, dinâmico e em permanente mobilidade; (2) concepção do sujeito constituído na inter-relação tensa e contraditória entre a subjetividade individual e a subjetividade social; (3) possibilidades de reconfiguração subjetiva e mudanças nos núcleos das configurações subjetivas diante de novos sentidos subjetivos produzidos; (4) produção contínua de sentidos subjetivos que se articulam às configurações subjetivas (González Rey, 2007; González Rey \& Autor 2, 2017; González Rey et al. 2017).

Produzir conhecimento no contexto da complexidade da sociedade contemporânea implica em resgatar o pesquisador - cientista - do lugar de tabulador e processador de dados para o lugar de produtor de conhecimento como resultado da articulação construção-interpretação no contexto teórico que sustenta o fenômeno estudado. A Epistemologia Qualitativa tem como pressuposto principal a legitimação do processo construtivo-interpretativo que leve à novos modelos teóricos em torno dos objetos em estudo. "O conhecimento legitima-se na sua continuidade e na sua capacidade de gerar novas zonas de inteligibilidade acerca do que é estudado" (González Rey, 2005a, p. 6).

$\mathrm{Na}$ implementação de uma pesquisa orientada pela Epistemologia Qualitativa o pesquisador necessita ser sujeito, dialogando, construindo, interpretando e confrontando informações, pois depende de seu poder criativo e imaginativo para explicar o fenômeno por meio de construções oriundas da articulação entre sua base teórica e 
as informações, no processo da pesquisa. Nesse processo, a legitimidade do conhecimento não existe em si mesma, mas está pautada pelo que representa o conhecimento produzido em termos da "ampliação do potencial heurístico da teoria, o qual permite acesso às áreas do real que resultavam inacessíveis em momentos anteriores” (González Rey, 2002, p. 135).

\section{A Metodologia Construtivo-Interpretativa}

A metodologia construtivo-interpretativa é um processo complexo e dinâmico de produção do conhecimento que envolve ativamente os participantes, incluindo o pesquisador e, embora o caráter construtivo e o caráter interpretativo sejam parte de um mesmo processo, em que um orienta e complementa o outro, possuem particularidades que merecem atenção especial (González Rey, 2015). O processo interpretativo é sempre a produção de um novo significado sobre informações e eventos que, em seu relacionamento, não tem significados a priori. A interpretação das informações ocorre ao longo de toda a pesquisa e vai alimentando novas construções no processo. Cada pesquisador constrói e reconstrói o problema de pesquisa tecido pelas suas vivencias e concepções sociais, históricas, culturais e, principalmente, epistemológicas, que é o que possibilita reconhecer a existência de um problema de pesquisa.

O caráter construtivo desafia a capacidade do pesquisador, tendo como referência sua base teórica, de produzir inteligibilidades em torno das informações geradas ao longo da pesquisa. As interpretações do pesquisador sobre informações e ventos constituem o que se denomina indicadores que não são mais que significados gerados pelo pesquisador que não estão explícitos no material empírico. A articulação de indicadores que apontam na mesma direção vai configurando hipóteses que, ao longo do processo investigativo, vão conformando o modelo teórico construído como resultado da pesquisa (González Rey, 2002, 2003, 2005a, 2005b, 2011, 2015; González Rey \& Autor 2, 2016, 2017).

Os instrumentos, no curso da pesquisa, são tomados como favorecedores da expressão do outro, representando uma fonte de produção da informação, e não categorias em si mesmos. A interlocução entre os instrumentos gera uma singularidade de informações que foge às regras padronizadas de produção de conhecimento, uma vez que requerem a participação interpretativa e construtiva do pesquisador ao longo de toda a pesquisa. Os instrumentos, mencionados a seguir, não foram utilizados de forma fragmentada e estanques, mas como fontes produtivas para a construção de indicadores no processo dialógico que caracteriza a pesquisa, na qual as dinâmicas conversacionais têm papel essencial como verdadeiras conversações entre pesquisador e participantes, diferentemente dos modelos centrados na lógica estímulo-resposta. Além dos instrumentos como favorecedores escritos e não escritos, utilizamos como 
fonte de informação os momentos informais, a análise documental e a observação, conforme veremos a seguir.

\section{Instrumentos apoiados em favorecedores não escritos}

Desenho Temático: Foi produzido como possibilidade de expressão de processos e formações subjetivas de forma diferente da palavra. Os participantes foram convidados a desenharem e, na sequência, descreverem oralmente a produção realizada: "(1) Minha maior alegria na escola (2) Minha maior tristeza na escola" (Rossato, 2009). Nesse momento, outras histórias, além da associada ao registro pictográfico também foram solicitadas e registradas pelo pesquisador.

Como eu me Sinto: Foi construído para o estudante expressar sua produção subjetiva diante de situações cotidianas da sala de aula. Todas as situações apresentadas foram vivenciadas pela pesquisadora nos momentos de observação, vividas por diferentes estudantes da turma. Foram produzidos dois materiais: 10 frases onde 0 estudante era levado, pela pesquisadora, a imaginar uma situação: "(1) Imagine que mesmo depois da professora explicar várias vezes um conteúdo você ainda não tenha entendido. Como você se sente? (2) Imagine que você perceba que está conseguindo fazer sozinho, atividades que não conseguia fazer. Como você se sente?" (Rossato, 2009); 10 cartões com expressões faciais, por meio de emotions, onde o estudante deveria escolher qual melhor representava seus sentimentos diante da situação escolhida e narrar histórias, igual ou semelhantes, vividas pelo mesmo, caso houvesse.

Mudando Você: Foi construído como um espaço para o estudante expressar alguma percepção negativa que tivesse em relação a si mesmo, por meio de uma história que narrava o encontro do estudante com um gênio e lhe concedia pedir três mudanças em si mesmo. "Imagine que você encontrasse um gênio e tivesse a chance de pedir três mudanças em você, mas teria que convencê-lo da importância dessas mudanças" (Rossato, 2009). O participante deveria escolher quais mudanças gostaria de fazer e argumentar oralmente a respeito de suas escolhas.

Escolhas: Instrumento construído para possibilitar a expressão da percepção de si, suas ações e as relações com o outro. Foram produzidos dois materiais nesse instrumento: 10 frases indutoras de escolhas que o estudante deveria fazer, utilizadas pelo pesquisador, como: "Quem você escolheria? (1) Para estudar junto (2) Para dividir seu lanche (3) Para ajudar a resolver problemas de matemática (4) Para ser seu melhor amigo" (Rossato, 2009). As escolhas deveriam ser feitas entre 30 cartões com imagens de pessoas (adultos, crianças, animações), todas dispostas em cima de uma mesa, no campo de visão do estudante. Após cada escolha, o estudante era levado a justificá-la, além de argumentar os motivos que o levaram a não escolher outros cartões para a realização da ação proposta. 
Complemento de Frases Ilustrado: Instrumento desenvolvido para identificar elementos que pudessem expressar a constituição da subjetividade individual em crianças. Foi realizado por meio de 27 cartões, produzidos pela pesquisadora, contendo frases que deveriam ser completadas oralmente. A atividade foi desenvolvida em forma de jogo, com regras criadas pelo próprio estudante. "(1) Eu fico feliz quando... (2) Os melhores momentos da minha vida são quando... (3) Eu me relaciono bem com... (4) É difícil aprender quando..." (Rossato, 2009).

Linha da Vida Escolar: O instrumento foi produzido para que o estudante reconstruísse sua trajetória escolar, demarcando os episódios que considerasse significativos em sua trajetória. Para a realização da tarefa, os estudantes receberam um papel (30x100) e a orientação para que dividissem pelo número de anos que já haviam frequentado a escola. Cada estudante construiu uma linha longitudinal no centro do papel perpassando todos os anos de escolaridade e, com os recursos de sua escolha (desenho, colagem, escrita, etc.), produziram cenas que foram posteriormente comentadas pelo mesmo.

\section{Instrumentos apoiados favorecedores escritos}

Redação: Foi desenvolvido para avaliar a expressão escrita do estudante, servindo de parâmetro para a produção dos demais instrumentos. Em uma folha de papel, foram solicitados a escreverem a partir da pergunta "Quem sou eu?" (Rossato, 2009).

Complemento de Frases: Instrumento criado por González Rey e Mitjáns Martínez (1989), com objetivo de identificar processos e formações configurados subjetivamente. Na pesquisa, foi utilizado com os professores, contendo 77 indutores curtos, diretos e indiretos. "(1) Eu gosto de... (2) O tempo mais feliz... (3) Gostaria de saber... (4) Eu aprendo... (5) Lamento...". Ao longo do instrumento foram introduzidos também alguns indutores diretos, relativos ao tema, como: "(11) Sou um professor... (19) Não esqueço da aula quando... (48) Gosto quando o aluno... (57) A sala de aula..." (Rossato, 2009). A proposta é de que a expressão seja livre, com primeira ideia que ocorrer ao participante.

Exploração múltipla: Orientado para o estudo dos indicadores funcionais como a flexibilidade e a capacidade de estruturação do campo de ação. Utilizou-se a adaptação feita por Mitjáns Martínez (2003). Na pesquisa, foi utilizado com os professores. É composto por seis expressões, como exemplificada, a seguir: "Se você pudesse escolher os alunos para compor sua turma, que alunos você escolheria? Faça uma lista das principais características dos alunos que você gostaria de ter em sua sala de aula".

Questionário Aberto: Os professores foram convidados a escreverem sobre: "Facilidades que o(a) aluno(a) encontra na escola - reação do(a) aluno(a); Dificuldades que o(a) aluno(a) encontra na escola - reação do(a) aluno(a); Impedimentos na 
aprendizagem do(a) aluno(a); Expectativa sobre sua participação na aprendizagem do(a) aluno(a); Impressões sobre as condições de trabalho oferecidas que visam a superação das dificuldades de aprendizagem do(a) aluno(a)" (Rossato, 2009).

Mudanças: Este instrumento foi desenvolvido para uma fase intermediária da pesquisa com o objetivo específico de identificar como o estudante percebia as mudanças ocorridas dentro do período em que a pesquisa estava sendo desenvolvida. Como material, utilizamos uma folha impressa com um quadro contendo cinco grupos sociais: - "(1) Minha Família (2) Minha Escola (3) Meus Amigos (4) Minha Professora (5) Minha Sala de Aula" (Rossato, 2009) - e duas colunas onde deveriam escrever sobre como percebiam cada um dos grupos em dois momentos: no ano passado e no momento presente.

Momentos informais. Ao longo da pesquisa muitas informações foram produzidas a partir dos momentos informais, de modo especial participando dos horários de intervalo das aulas juntamente com os professores. Os momentos informais tanto podem ser os criados a partir das relações espontâneas com os participantes como os decorrentes da interação com os instrumentos da pesquisa, pelos indutores diretos e indiretos ali contidos.

Análise Documental. Os documentos foram utilizados como fonte de informação na caracterização dos participantes, pois contém o registro da história escolar. Vale lembrar que essas informações pontuais contribuem para que o pesquisador possa compreender melhor as expressões do estudante e possa identificar a descrição de fatos, na versão do outro. Foram analisados a pasta com os documentos do estudante, os relatórios bimestrais do estudante, os cadernos de sala de aula, as fichas do conselho de classe

Observação. Permite identificar comportamentos intencionais ou não-intencionais em seu contexto temporal-espacial, independentemente da capacidade verbal expressiva dos participantes, possibilitando o confronto com informações obtidas por meio dos instrumentos. A observação foi utilizada como recurso para perceber o movimento dos sujeitos na relação com o outro (professores expressando suas opiniões sobre os estudantes, professores conversando com estudantes, reação dos estudantes diante da postura assumida pelo professor a seu respeito, reação dos estudantes diante da postura assumida pelo professor diante dos colegas, reação dos estudantes na ausência dos professores, reação dos estudantes junto aos colegas da escola, estudantes expressando sua opinião sobre os professores, etc.), levantando e reforçando indicadores formulados no processo da pesquisa. Foram observadas as dinâmicas do conselho de classe, das reuniões pedagógicas, os momentos de coordenação pedagógica, os intervalos das aulas e as aulas. 


\section{O processo de produção das informações no decorrer da pesquisa}

Seleção dos Participantes. Os possíveis participantes da pesquisa, inicialmente, foram apontados pelos professores e, posteriormente, analisados dentro do perfil necessário à pesquisa: estudantes que apresentavam dificuldades de aprendizagem sem nenhuma causa orgânica. Nessa abordagem inicial chegou-se a 30 (trinta) estudantes que, durante 3 (três) semanas, foram acompanhados no contexto da escola. Depois dessa fase inicial, optou-se por continuar com 17 (dezessete) estudantes que demonstraram ser mais significativos. Sobre os que deixaram o grupo: 6 (seis) estudantes apresentavam problemas relacionados ao comportamento, sem que fosse verificado interferência significativa na qualidade da aprendizagem; 4 (quatro) estudantes eram membros de uma comunidade indígena e, além das dificuldades com a língua, haveriam problemas burocráticos para conseguir autorização para a participação na pesquisa; 3 (três) estudantes foram transferidos de turno inviabilizando a continuidade no grupo.

Identificação e Análise da Constituição Subjetiva dos Estudantes. A segunda etapa da pesquisa foi marcada pelo trabalho direto com os 17 (dezessete) estudantes selecionados, para identificar como estava constituída a subjetividade. Foram produzidos indicadores iniciais dos processos e formações subjetivos que poderiam contribuir para explicar as dificuldades de aprendizagem escolar. Essa etapa teve duração de aproximadamente 7 (sete) meses.

O Movimento da Subjetividade. Na etapa final da pesquisa trabalhamos com 5 (cinco) estudantes que, após um ano de investigação, apresentaram melhora no desempenho escolar qualitativamente significativa para a investigação. Destaca-se que a pesquisa não fez nenhuma abordagem interventiva em relação às dificuldades de aprendizagem, apenas acompanhou a dinâmica da escola e o trabalho que os professores foram desenvolvendo com os estudantes. Essa etapa final teve duração de 6 (seis) meses.

Os 12 (doze) meses de atividades descritas na Primeira Etapa e Segunda Etapa possibilitaram criar uma base de informações e indicadores que, confrontados com a produção da Terceira Etapa, geraram um conteúdo passível de análise do movimento mudança e desenvolvimento - da subjetividade. Foram produzidas informações sobre os significados produzidos pelos professores em relação às mudanças ocorridas nos estudantes, tanto em relação à aprendizagem escolar como em relação às mudanças subjetivas, expressas na mudança do comportamento aprendente, bem como sobre como a escola está configurada subjetivamente no estudante destacando elementos que contribuíram com a superação das dificuldades de aprendizagem escolar e com as mudanças subjetivas no estudante. 


\section{Análise dos resultados: o processo construtivo-interpretativo}

No modelo construtivo-interpretativa, a ação do pesquisador com as informações é realizada ao longo de toda a pesquisa, pois é o que lhe possibilita ir tecendo o caminho da mesma, balizado pela qualidade e pertinência dos indicadores produzidos para o problema em discussão e, principalmente, para a abertura de novos campos de inteligibilidade dentro do modelo teórico em discussão. A interpretação e a construção do pesquisador requerem um olhar sensível, capaz de se movimentar na tessitura das informações desde as mais particulares até as mais gerais sem, contudo, ficar preso aos fatos narrados e descritos pelos participantes, mas aproximando-se dos processos e formações subjetivos produzidos nas vivências dos mesmos, sempre fruto da interpretação qualificada e fundamentada pela base teórica do pesquisador.

$\mathrm{Na}$ pesquisa desenvolvida, o processo de interpretação das informações ocorreu ao longo de todo o período de investigação, em quatro níveis inter-relacionados. No primeiro nível realizou-se a produção de indicadores sobre a constituição da subjetividade dos estudantes. Essa produção é um exercício interpretativo do pesquisador, construído pela tessitura da análise integrada das informações. Os indicadores podem ir ganhando força ou mesmo serem refutados ao longo da pesquisa, sempre em diálogo com o conjunto das informações produzidas no próprio processo da pesquisa.

No segundo nível, a partir dos indicadores mais consistentes, foram produzidas hipóteses sobre como a constituição da subjetividade se expressava nas dificuldades de aprendizagem e, ao longo da pesquisa, no processo de superação das dificuldades de aprendizagem. As pesquisas regidas pela Epistemologia Qualitativa visam contribuir tanto na produção de novos campos de inteligibilidade sobre o problema estudado, como para a produção de novas zonas de sentido à base teórica do pesquisador.

Os novos campos de inteligibilidade constituem um terceiro nível construtivo-interpretativo em que são destacadas as contribuições relacionadas ao objeto de pesquisa como novas possibilidades de compreensão do problema. No caso da pesquisa desenvolvida, o desenvolvimento da subjetividade foi pesquisa por meio dos processos de superação das dificuldades de aprendizagem, levando-nos à uma profunda problematização e reflexão sobre o tema. Como resultado desse processo, foi possível uma sistematização de novas inteligibilidades sobre o mesmo.

Uma vez percorrido esse caminho, no quarto nível do processo construtivo- interpretativo, foram produzidas as novas zonas de sentido relacionadas às contribuições da pesquisa ao modelo teórico em construção - teoria da subjetividade na perspectiva cultural-histórica. Pelas limitações de espaço do artigo, a seguir, destacaremos brevemente somente a produção relativa ao terceiro e quarto nível de análise. O primeiro e segundo nível, pela densidade descritiva dos mesmos, poderão ser consultados em Rossato (2009). 


\section{Novos campos de inteligibilidade e zonas de sentido}

A pesquisa por meio da metodologia construtivo-interpretativa nos possibilitou construir três novos campos de inteligibilidade sobre as dificuldades de aprendizagem. O primeiro refere-se às dificuldades de aprendizagem escolar gerada pela negação do sujeito do aprender. Na escola, a negação do sujeito em detrimento do modelo clássico de aluno tem sido registrada como parte da história educacional em nosso país. Em nossa pesquisa, registramos que essa negação do sujeito do aprender pode ser produzida, também, por outros espaços sociais, como a família, que, muitas vezes, reproduz os modelos da escola. O estudante, ao ter sua expressão singular da subjetividade negada no espaço escolar, tem negada suas possibilidades de constituir-se sujeito de sua própria aprendizagem e desenvolvimento. Em outras palavras, tira-se do estudante a possibilidade de uma produção de sentidos subjetivos que possibilitaria que sua aprendizagem se constituísse como uma configuração subjetiva eu favorecesse o próprio processo de aprendizagem.

O segundo campo de inteligibilidade refere-se às dificuldades geradas pela ausência de condições favorecedoras à produção de sentidos subjetivos que promovam a aprendizagem escolar. Toda ação e relação que gere emocionalidade é produtora de sentido subjetivo. O que acontece, muitas vezes, é que esse sentido subjetivo produzido na ação e relação que envolve a aprendizagem não é favorável à ocorrência da mesma. Ao reconhecermos a dimensão subjetiva da aprendizagem escolar, as dificuldades deixam de ser vistas como resultado do intelecto ou do orgânico e passam a ser compreendidas como expressão de processos subjetivos de natureza social.

O terceiro campo de inteligibilidade refere-se às dificuldades de aprendizagem geradas pela existência de configurações subjetivas geradoras de danos que comprometeriam a produção de sentidos subjetivos do aprender. Identificar quais são as configurações subjetivas envolvidas na produção dos sentidos subjetivos gerados na aprendizagem escolar não é uma tarefa fácil, muito menos detectar como participam da produção das dificuldades de aprendizagem escolar. Estamos demarcando a existência de configurações subjetivas qualitativamente diferenciadas que "participam na ação pelos sentidos subjetivos e, nessa condição, são parte da emergência de novos sentidos subjetivos que podem chegar a modificar as próprias configurações do sujeito implicado nessa atividade" (González Rey, 2007, p. 159).

A metodologia construtivo-interpretativa possibilitou, por fim, a construção de duas novas zonas de sentido dentro do modelo teórico utilizado na pesquisa, de modo especial relativas ao desenvolvimento da subjetividade. Em primeiro lugar, a constituição do sujeito no confronto com o outro. A produção dos sentidos subjetivos segue desdobramentos e entrelaçamentos que nunca são universais, mas que posicionam a pessoa como sujeito de seu próprio desenvolvimento e, nesse sentido, o outro tem 
importância para o desenvolvimento quando se converte em uma fonte de produção de sentido subjetivo, numa relação dialógica. A simples presença do outro não garante a produção de sentido subjetivo, ao contrário, o outro tem importância no desenvolvimento pela qualidade dos sentidos subjetivos que é capaz de produzir, pois os sentidos subjetivos se produzem na geração do espaço simbólico-emocional particular em cada sujeito. "O outro é significativo para o desenvolvimento ao converter-se em uma fonte de sentido subjetivo" (González Rey, 2004, p. 20). Essa afirmação do autor sustenta nossas considerações anteriores, porém destacamos que o desenvolvimento só ocorre quando essa produção de sentido subjetivo não é passageira, ou seja, quando ela é capaz integrar a organização das configurações subjetivas e gerar novos caminhos para que outras mudanças aconteçam. O outro tem importante papel no desenvolvimento humano, pois tanto pode abrir como fechar os espaços de mobilidade do sujeito pela emocionalidade gerada nas relações produtoras de sentidos subjetivos. As configurações subjetivas individuais e dos espaços sociais entram de forma entrelaçada na produção da emocionalidade, que, pela via dos sentidos subjetivos produzidos, atuam na constituição do sujeito.

Em segundo lugar, o desenvolvimento da subjetividade integrado à mudança na personalidade gerada pelas reconfigurações subjetivas e produção de novos sentidos subjetivos. A personalidade é o sistema subjetivo auto-organizador da experiência do sujeito, constituída em sua história, o que lhe atribui um caráter temporal e com mobilidade (González Rey, 1995), e somente com essa compreensão de personalidade podemos, efetivamente, pensar o sujeito em permanente processo de mudança, sem ter que carregar para toda vida os rótulos sociais criados em função das condições vividas em determinadas circunstâncias de sua história. A produção de novos sentidos subjetivos, tomada na centralidade do processo de desenvolvimento da subjetividade, apresenta-se como uma possibilidade de rompimento com o determinismo humano e com a personalidade concebida como um conjunto de elementos fixos. Embora sujeito e personalidade sejam constituidores da subjetividade individual, a subjetividade social não está isenta nesse processo, ao contrário, o sujeito se integra à subjetividade social gerando novos focos de subjetivação, não de forma intencional e direta, mas como um momento no movimento de um sistema complexo, que não responde de maneira direta nem linear à intencionalidade humana. Esse confronto do sujeito com a subjetividade social traduz-se num espaço gerador de conteúdos simbólico-emocionais produtores de sentidos subjetivos, demarcando a constituição do sujeito numa matriz relacional.

\section{Considerações finais}

A metodologia construtiva-interpretativa possibilitou reconhecer a singularidade de cada caso, promovendo rupturas com a tradição psicológica de generalização 
dos processos e problemas vivenciados pelos estudantes no contexto escolar. Além disso, os casos apresentados permitiram gerar inteligibilidade acerca da diversidade do singular nas dinâmicas relacionais envolvidas na aprendizagem escolar, além de compreender a pesquisa qualitativa como um processo de construção dinâmico de configurações subjetivas diferentes, recuperando a dimensão subjetiva do sujeito que aprende, que aparece primeiro como sujeito no diálogo com o outro, mudando o seu posicionamento social na escola e mobilizando um processo de desenvolvimento subjetivo que implicará avanços no aprender.

Outro ponto de destaque na pesquisa sobre o desenvolvimento da subjetividade foi o papel do pesquisador que precisou converter-se também em sujeito na pesquisa e parte da realidade investigada. A ação ativa e criativa do pesquisador, numa relação dialógica com os participantes, produziu um espaço dinâmico-comunicacional favorável para a expressão dos sujeitos - pesquisador e participante -, condição essencial para a produção de informações no contexto da metodologia construtivo-interpretativa.

Por fim, por meio da pesquisa desenvolvida, o desenvolvimento da subjetividade pode ser caracterizado como mudanças subjetivas que ganham certa estabilidade, originando outras mudanças, gerando novos níveis qualitativos de organização subjetiva (Rossato, 2009; Rossato \& Mitjáns Martinez, 2013; 2015). Pesquisar, nesse movimento, sem a pretensão de congelar a cena para que possa ser analisada, coloca os pesquisadores diante do desafio da produção do conhecimento com fenômenos complexos, reconhecendo que não há uma estabilidade que possa ser retratada, imprimindo dinamicidade e complexidade teórico-epistemológica-metodológica à pesquisa.

\section{Referências}

González Rey, F. (2015). Ideias e modelos teóricos na pesquisa construtivo-interpretativa. In A. Mitjáns Martinez; M. Neubern \& V. D. Mori (Orgs.). Subjetividade contemporânea: discussões epistemológicas e metodológicas. (pp.13-34). Campinas SP: Alínea Editora.

González Rey, F. (2013). O que oculta o silêncio epistemológico da Psicologia? Pesquisas e Práticas Psicossociais, 8(1), pp. 20-34. https://www.ufsj.edu.br/portal2-repositorio/File/revistalapip/ Volume8_n1/PPP-8Abstract-Art_2.pdf.

González Rey, F. (2007). Psicoterapia, subjetividade e pós-modernidade. Uma aproximação históricocultural. São Paulo: Pioneira Thompsn Learning.

González Rey, F. (2005a). O valor heurístico da subjetividade na investigação psicológica. In F. González Rey (Org.). Subjetividade, complexidade e pesquisa em psicologia. (pp. 27-51). São Paulo: Pioneira Thomson Learning (pp. 27-51).

González Rey, F. (2005b). Pesquisa Qualitativa e Subjetividade: os processos de construção da informação. São Paulo: Pioneira Thomson Learning. 
González Rey, F. (2004). O sujeito, a subjetividade e o outro na dialética complexa do desenvolvimento humano. In L. M. Simão \& A. Mitjáns Martinez (Orgs.) O outro no desenvolvimento humano. (pp. 1-28). São Paulo: Pioneira Thompsn Learning.

González Rey, F. (2003). Epistemología Cualitativa y Subjetividad. São Paulo: EDUC.

González Rey, F. (2002). Pesquisa Qualitativa em Psicología: caminhos e desafios. São Paulo: Pioneira Thompson Learning.

González Rey, F. (1995). Comunicación, personalidad y desarrollo. Ciudad de Habana, Cuba: Editorial Pueblo y Educación.

González Rey, F. \& Mitjáns Martinez, A. (2017). Subjetividade: teoria, epistemologia e método. Campinas: Alínea.

González Rey, F. \& Mitjáns Martinez, A. (2016). Una epistemologia para el estudio de la subjetividad: sus implicaciones metodológicas. Psicoperspectivas (Online): Individuo y Sociedad, 15(1), pp. 5-16. http://www.psicoperspectivas.cl/index.php/psicoperspectivas/article/view/667

González Rey, F. \& Mitjáns Martinez, A. (1989). La personalidad su educación y desarrollo. Ciudad de La Habana, Cuba: Editorial Pueblo y Educación.

González Rey, F; Mitjáns Martinez, A.; Rossato, M. \& Goulart, D. M. (2017). The relevance of the concept of subjective configuration in discussing human development. In: M. Fleer; F. González Rey \& N. Veresov. Cultural-historical perspectives on Emotions: Advancing the concepts of perezhivanie and subjectivity. (pp. 217-243). Singapore: Springer.

Rossato, M. (2009). O movimento da subjetividade no processo de superação das dificuldades de aprendizagem escolar. Tese de Doutorado. Brasília: Universidade de Brasília.

Rossato, M. \& Mitjáns Martinez, A. (2017). A metodologia construtivo-interpretativa como expressão da Epistemologia Qualitativa na pesquisa sobre o desenvolvimento da subjetividade. In A. P. Costa; P. A. Castro; S. O. Sá \& R. A. Saavedra (Eds.), $6^{\circ}$ Congresso Ibero-Americano em Investigação Qualitativa. Investigação Qualitativa na Educação (pp. 343-352). Aveiro, Portugal: Ludomedia. http://proceedings.ciaiq.org/index.php/ciaiq2017/article/view/1352/1310

Rossato, M. \& Mitjáns Martinez, A. (2015). A subjetividade dos estudantes no processo de superação das dificuldades de aprendizagem: estratégias de ação. In A. A. Anache, B. J. L. Scoz \& M. I. S. Castanho (Orgs.). Sociedade Contemporânea: subjetividade e educação. (pp. 33-53). São Paulo: Menon.

Rossato, M. \& Mitjáns Martinez, A. (2013). Desenvolvimento da subjetividade: Análise de histórias de superação das dificuldades de aprendizagem. Revista Semestral da Associação Brasileira de Psicologia Escolar e Educacional, 17(2), pp. 290-297. http://www.scielo.br/pdf/pee/v17n2/ v17n2a11.pdf

Santos, B. S. \& Meneses, M. P. (Orgs.). (2010). Epistemologias do Sul. São Paulo: Cortez. 


\section{Maristela Rossato}

Programa de Pós-Graduação Processos de Desenvolvimento Humano, Departamento de Psicologia Escolar e Desenvolvimento, Instituto de

Psicologia, Universidade de Brasília, Brasília, Brasil

Email: maristelarossato@gmail.com https://orcid.org/0000-0001-6457-9005

Albertina Mitjáns Martinez

Programa de Pós-Graduação em Educação, Faculdade de Educação, Universidade de Brasília, Brasília, Brasil

Email: amitjans49@gmail.com https://orcid.org/0000-0003-3094-2886

\section{Correspondência}

Maristela Rossato

Universidade de Brasília, ICC-Sul, Instituto de Psicologia, Departamento de

Psicologia Escolar e Desenvolvimento

Asa Norte. Brasília, Brasil

CEP 70910-900

Data de submissão: Março de 2017

Data de avaliação: Abril de 2017

Data de publicação: Julho 2018 